\title{
Zu Besuch bei einer Enkelin Felix Mendelssohns*
}

Von Heinz Balmer

Mein Heimatort Wilderswil bewahrt Exzellenz Adolf Wach in gutem Andenken. Der alte Geheimrat hat viel für das Dorf getan. Er lebte 1843 bis 1926. Ein Meister des Zivilprozeßrechtes, lehrte er von 1875 an als Ordinarius in Leipzig. Seine Frau Lili war die jüngste Tochter Felix Mendelssohns.

Von dem Tondichter her stammte die Verbundenheit mit dem Berner Oberland. Schon vor 1880 verbrachte die Familie Wach jeden Sommer ihre Ferien in einem Hotel in Wilderswil. Als eines Tages die Alp Ried versteigert wurde, ließ sich Professor Wach von Einheimischen nach längerem Zureden bewegen, sie zu erwerben. Im Frühling 1964 lebte dort noch seine Tochter Marie Wach.

Fräulein Heidi von Steiger von Goldswil war bereit, meine Frau und mich hinzuführen. Sie holte uns mit ihrem kleinen weißen Auto am Bahnhof Interlaken $\mathrm{Ost}$ ab und erzählte uns unterwegs, daß dort, wo die Alp beginne, drei Chalets entstanden seien. Wach ließ sein Haus ganz von Wilderswiler Handwerkern errichten. Später kam ein Pächterhaus dazu, und noch später baute der älteste Sohn Felix Wach das dritte Haus. Verwundert hörten wir, daß Fräulein Wach kein elektrisches Licht habe; auch gekocht werde nach alter Väter Sitte. Allein könnte sie jetzt, mit 87 Jahren (geboren am 8. Mai 1877), im Vaterhause nicht mehr wohnen. Doch habe sie einst eine Kriegswaise aufgenommen. Inge sei bei ihr geblieben und betreue sie gut. Fräulein Wach sei gegen Kälte abgehärtet. Andere Leute bekämen in der Wohnung, die nur durch einen Trittofen heizbar sei, im Winter bestimmt eine Lungenentzündung.

Während dieses Gesprächs erreichten wir Wilderswil, schwenkten in das Sträßchen nach Saxeten ab und fuhren durch den Wald hinauf. Gleich oben am Wald bogen wir links durch einen hölzernen Torbogen ein und hielten auf dem Platz bei den Chaletbauten an.

Es regnete. Fräulein Wach hatte den Wagen gehört. Auf der Laube über

\footnotetext{
* Nach Notizen ausgearbeitet für Herrn Prof. Huldrych M. Koelbing zum 60. Geburtstag. Ihm verdanke ich mein Wirken in der Schweiz. Und ihm möchte ich mit diesem Beitrag dafür danken, daß er, über das Medizingeschichtliche hinaus, mit jeder Art der Kunst verbunden und für Kulturgeschichtliches empfänglich ist.
}

Gesnerus 1/2 (1983) 
uns öffnete sich die Türe; Fräulein Wach trat an das Geländer heraus und grüßte freundlich herunter. Ich erblickte eine kleine, zierliche Greisin. Ihr Anblick und ihr Gehstock mit dem altertümlichen Handgriff erinnerten mich irgendwie an Friedrich den Großen.

Wir stiegen die Treppe hinauf und betraten eine große Stube. Eine Petrollampe hing über vier Gedecken mit alten Täßchen und Tellern. Durch die Fenster sah man weit hinunter auf Interlaken und den Thuner- und Brienzersee. An der linken $W$ and stand ein außerordentlich breiter, uralter Schrank mit herrlichen Eisenbeschlägen; er stammte aus dem Kloster Interlaken. Zwischen der Eintrittspforte und diesem Schrank, auf dem alten Büfett, lehnten hundertjährige große Teller, die dunkelbraun und mit Blumen bemalt waren. Das kaminartige Holzgehäuse auf der rechten Seite barg den Aufzug für die Speisen aus der unten gelegenen Küche. Vorn gegen die Fenster zu hingen alte Stiche von Interlaken. Durch eine Türe gelangte man in ein zweites großes Zimmer, worin ein wunderbares Frauenbildnis hing. Wir erkannten Cécile Jeanrenaud, die Gattin Felix Mendelssohns; sie war eine Schweizerin. Ein kleines Sofa stand an der Wand; doch schlief Fräulein Wach nicht hier, sondern im oberen Stock. Hinten hinaus kam man auf eine Laube mit Korbstühlen. Im Hof rauschte ein Brunnen. Fräulein Wach deutete an, er sei aus Goldswiler Steinplatten gehauen. Dahinter erhob sich das dritte Chalet; es war durch einen gedeckten Treppengang mit dem Haus, in dem wir standen, verbunden.

Zunächst aber wurden wir nun im Raum mit dem zubereiteten Tische bewirtet. Es rumpelte im Aufzug, und als Fräulein Wach ihn öffnete, zeigten sich ein goldgelber Gebäckring, Gleichschwer genannt, und eine Kanne mit heißem Tee. Meine Frau und ich durften uns mit dem Blick nach den Fenstern setzen. Aber wir schauten wenig hinaus. Das Gesicht der Gastgeberin zog uns an. Unter der feinen Stirne strahlten blaue Augen. Schlicht war die Kleidung, geistvoll die Rede; es war für Fräulein Wach ganz natürlich, eine lateinische Wendung einzuflechten. Wir sprachen Mundart, wechselten aber unvermerkt ins Hochdeutsche hinüber. Nur Fräulein von Steiger blieb beim Berndeutschen. Sie nannte Fräulein Wach Tante, und ich erkundigte mich nach dem Grund.

Da gab Fräulein Wach kurz Auskunft über ihre Familie. (Mendelssohn selbst hatte fünf Kinder: Carl 1838, Marie 1839, Paul 1841, Felix 1843, Lili 1845. Felix starb mit acht Jahren. Carl wurde Professor der Geschichte in Heidelberg und Freiburg, Paul Farbstoffchemiker und Gründer der Agfa, Marie die Mutter des Oxforder Philologen Paul Benecke.) Das Ehepaar 
Adolf und Lili Wach in Leipzig hatte sechs Kinder: Felix, Hugo, Elisabeth, Dora, Marie und Adolf. Felix wurde wie sein Vater Professor der Rechte und Geheimrat in Leipzig; sein Sohn Joachim wirkte nach der Auswanderung als Professor für vergleichende Religionswissenschaften in Chicago. Hugo war zeichnerisch begabt und wurde Architekt. Elisabeth war mit Pfarrer Friedrich von Steiger in Hindelbank vermählt und somit die Tante von Fräulein von Steiger. Dora heiratete ihren Cousin, Carls Sohn Albrecht Mendelssohn, der als Geschichtsprofessor von Hamburg nach Oxford wechselte und als Engländer starb. Marie blieb nach dem ziemlich frühen Tode der Mutter beim Vater. Fräulein von Steiger warf ein, sie male schöne Aquarelle; aber Marie wehrte ab und erklärte, sie komme gar nicht mehr dazu. Adolf sei zwölf Jahre jünger und lebe als Anwalt mit einem Sohn Thomas in Zürich, habe aber vor einem Jahr einen Schlaganfall erlitten. (Kurzbiographie und Porträt siehe in: Schweizer biographisches Archiv, Band 6, Zürich 1958, S. 116.)

Zweimal wurden wir beim Essen von Besuchern überrascht. Zuerst drückte sich eine Katze zur Türe herein. Fräulein von Steiger fütterte sie auf dem Ofentritt mit Backwerk. Dann stürmten zwei kleine Buben heran, offenbar Söhne des Pächters. Als sie uns gewahrten, wurden sie scheu und verschwanden wieder.

Ich äußerte, meine ebenfalls 87jährige Großmutter erinnere sich, als Mädchen ins Ried gekommen zu sein, um zu singen. Fräulein Wach bestätigte, die Wilderswiler Schulkinder seien jedes Jahr zum Singen erschienen; dann seien sie bewirtet worden, und der Vater habe jedem 20 Rappen gegeben. Marie W ach hatte Sonntagsschule gehalten und besonders eine Nähschule für die Töchter des Dorfes betrieben. Bis 1933 hatte sie im Winter in Leipzig und im Sommer in Wilderswil gewohnt. Als sie 1934 ganz nach dem Ried zog, bestand die Nähschule noch.

Inge sahen wir nicht, denn sie hatte zwei andere Gäste zu versorgen: Christian, den Fräulein Wach gegenwärtig als Waldarbeiter angestellt hatte, und dessen Bruder, der heute aus Wilderswil zu ihm gekommen war und mit ihm in der Küche saß. Fräulein Wach fügte bei, mit Inge habe sie das große Los gezogen.

Nun sprachen wir uns auf Verwandte an. In Wilderswil gibt es viele Balmer. Fräulein Wach erinnerte sich, ihr Vater habe die Söhne des Statthalters Kaspar Balmer im Weiher auf dem Ried schwimmen gelehrt. Auch die Pächter auf dem Ried hätten Balmer geheißen; einer sei 93jährig geworden. Ich wußte nichts von all diesen. 
Auf die Verwandtschaft mit ihrem Großvater Mendelssohn bildete sich Fräulein Wach nichts ein. Man unterscheide Wahl-, Qual- und Prahlverwandte. Meine Frage, ob sie musikalisch sei, verneinte sie. Fräulein von Steiger erhob lebhaft Einspruch; aber Marie Wach erklärte, wenn jemand dies frage, erwarte er mehr. Sie und ihre Geschwister hätten die Gabe nicht gehabt; nur ein entfernter Vetter, Arnold Mendelssohn, habe etwas davon besessen.

In Leipzig hatte sie den alten Brahms gekannt; er sei aber nie im Ried gewesen. Als nahen Freund des Hauses erwähnte sie den Komponisten Heinrich von Herzogenberg. Es sei merkwürdig, daß man ihn so wenig kenne.

Marie Wach schien noch das Leben des letzten Jahrhunderts zu führen. «Elektrisch ist verboten!», meinte sie schalkhaft. Es gefiel ihr, daß wir kein Auto hatten. Fräulein von Steiger verteidigte sich, über das «Goldswiler Taxi» sei Fräulein Wach schon oft froh gewesen.

Nachdem wir das Geschirr in den Aufzug gestellt hatten, fragte ich nach dem Klavier und vernahm, das Musikzimmer sei unten im andern Chalet. Fräulein Wach schritt sofort zur Laube und über die Treppen des Verbindungsganges. Hinter ihr gehend, betrachtete ich die zarte, gebrechlich anmutende Gestalt. Ihre Finger waren blaurot, die Beine O-förmig, der Rücken bucklig und eine Schulter schief. Die beiden letzten Eigenschaften sind seit dem Großvater von Felix, dem Philosophen Moses Mendelssohn, dem Urbild «Nathans des Weisen», von einzelnen Familienmitgliedern bezeugt.

Am jenseitigen Hause stiegen wir wieder eine lange Treppe hinab, und Fräulein Wach drückte die Falle einer nie verschlossenen Türe nieder. Fräulein von Steiger half mit einem Rucke nach, und wir betraten den Musiksaal. Im Dämmer stand ein schwarzes Klavier. «Das hat uns Reger ausgewählt», sagte Fräulein Wach so schlicht, als ob das oft vorkäme. Der Klavierstuhl hatte einen viereckigen Rahmen mit Holzschnitzerei; als Polster lag darin ein rotseidenes Kissen mit drei Rosen. Dieser Stuhl war ein Geschenk Goethes an den zwölfjährigen Felix. Auf einem Sockel in der Ecke stand das Original einer wundervollen Marmorbüste, die der Bildhauer Ernst Rietschel nach der Totenmaske Mendelssohns gemeißelt hatte. Als Lili, damals ein ganz kleines Mädchen, der Büste ansichtig wurde, habe sie gejubelt: «Der Papa ist wieder da!» Dies zeuge wohl für ihre Ähnlichkeit. Im Hintergrund gewahrten wir ein schönes Ölbildnis Mendelssohns vom gleichen Künstler, der auch Cécile Jeanrenaud gemalt hatte. Es war Eduard 
Magnus. (Über dessen Bruder, den Physiker Gustav Magnus, hat sein Schüler und Nachfolger Hermann Helmholtz 1871 eine seiner Reden gehalten.)

Fräulein Wach berichtete, daß Clara Schumann auf dem Klavier geübt und vorgespielt habe. Einige Männer von Wilderswil hätten sie auf einem Tragstuhl gebracht. Ein solcher Sitz war zu beiden Seiten wie eine Bahre mit Holmen versehen, und es war ein Erwerbszweig, Ausländer damit bergan zu schleppen. Auf der damaligen Rechnung habe es geheißen: «Ein altes $\mathrm{W}_{y b}$ ins Ried hinaufgebracht».

Wir kehrten in das Zimmer mit dem Gemälde der Cécile Jeanrenaud zurück. Dort zeigte uns Fräulein Wach am Schreibtisch die Bilder ihrer Eltern. «Alles ist alt hier», betonte sie. Die Standuhr versah ihren Dienst seit 200 Jahren. Auch die vielen gebundenen Bücher mit ihren Rückenschildchen, in Gestellen nebenan und hier, schienen alt.

Wir dachten an den Aufbruch, da Fräulein Wach von einer kürzlichen Reise nach Zürich und Winterthur vielleicht müde war. Doch sie selber fragte uns, ob wir gerne noch Aquarelle ihres Großvaters ansehen möchten, und trug ein großformatiges Buch herbei. Wir durften, am Tische sitzend, darin blättern. Ich hatte nicht gewußt, daß Felix Mendelssohn vollendet schöne farbige Aquarelle geschaffen hatte. Mit Recht bemerkte Fräulein W ach: «Ohne eine Spur von Kitsch.» Die Bilder waren, immer rechtsliegend, an ihrem Rande eingeklebt und mit zwei feinen Tuschestrichen umrahmt. Mendelssohn hatte das Buch kurz vor seinem Tode so gestaltet. Hinten auf den Zeichnungsblättern hatte er mit Bleistift Ort und Entstehungstag vermerkt. Alle Aquarelle waren vom Juni bis September 1847 auf einer letzten Schweizerreise entstanden, oft im Abstand weniger Tage. Fräulein Wach erläuterte, Mendelssohn habe geschwankt, ob er Musiker oder Maler werden solle, aber der Drang zur Musik sei elementarer gewesen. Die beiden ersten Ansichten zeigten den Rheinfall, die weiteren Bilder Luzern, Thun, einen Nußbaum, Unterseen mit dem Harder, den Höheweg in Interlaken, Mürren, Alpengipfel, einen Bergsee. Einige der Aquarelle waren nicht ganz fertig, und man sah die Vorzeichnung, zum Beispiel eines Graskarrens, in feinen, sicheren Strichen.

Fräulein Wach erzählte, daß die Briefe und Tagebücher Mendelssohns nun, da sie und ihr Bruder Adolf alt und gebrechlich geworden seien, in die Bodleian Library in Oxford übergegangen seien. Nur dieses Aquarellbuch habe sie bis zu ihrem Tode zurückbehalten wollen. Peter Sutermeister habe 1958 Mendelssohns «Briefe einer Reise durch Deutschland, Italien und die 
Schweiz» von 1830-1831 im Verlag von Max Niehans in Zürich herausgegeben. Er habe zur Herstellung der Briefabschriften fünf Wochen auf dem Ried geweilt und gerade damals die Stelle als Sekretär beim Nationalfonds angeboten erhalten.

Als ich bemerkte, ich hätte die kleinen Veröffentlichungen der Internationalen Mendelssohn-Gesellschaft gesehen, mahnte sie mit erhobenem Finger: «Davor ist zu warnen!» Jene Gesellschaft bestehe nur aus zwei Herren und gebe allerlei aus dem Papierkorb heraus. Die Hefte dünken mich trotzdem verdienstlich. Eines enthält Landschaftszeichnungen, eines Bildnisse und eines ein längeres Gedicht Mendelssohns in Hexametern, worin der Knabe in homerischem Stil die kriegerischen Erlebnisse von Spielgefährten köstlich schildert. Bei Mendelssohn ist eben sogar das Beiläufige ungewöhnlich.

Zuletzt kamen wir auf seine Lieder zu sprechen. In Leipzig habe der Chor der Thomaner immer zum Jahresanfang Mendelssohns «Neujahrslied» gesungen. Fräulein Wach sagte die Worte von Johann Peter Hebel auswendig vor:

\author{
«Mit der Freude zieht der Schmerz \\ Traulich durch die Zeiten, \\ Schwere Stürme, milde Weste, \\ Bange Sorgen, frohe Feste \\ Wandeln sich zur Seiten ... \\ Gebe denn, der über uns \\ Wägt mit rechter Waage, \\ Jedem Sinn für seine Freuden, \\ Jedem Mut für seine Leiden \\ In die neuen Tage.»
}

Fräulein Wach schien nicht ermüdet, als sie uns zum Abschied die Hand reichte. Aber bei der Rückfahrt gestand uns Fräulein von Steiger, daß sie nicht ohne Sorge sein könne. Schon vor zwei Jahren sei Fräulein Wach eine Treppe hinabgestürzt, habe aber zum Glück nichts gebrochen. Vor einiger Zeit sei sie am späteren Abend, als die Pächtersleute schon schliefen, beim Brunnen hinter dem Haus umgefallen. Sie behaupte zwar, nur ausgeglitten 
zu sein, aber jedenfalls sei sie nachher am Boden gelegen. Um niemanden zu wecken, sei sie auf allen Vieren die Treppen hinangekrochen. Im Schlafzimmer habe sie gemerkt, daß sie am Hinterkopf blutete, und habe den Schwamm, mit dem sie sich zu waschen pflege, mit einem Tuch hinten an den Kopf gebunden und so geschlafen. Inge habe ihr am Morgen eine halbe Stunde verkrustetes Blut aus den Haaren gewaschen.

Der Gefährdung stehe allerdings die Zähigkeit gegenüber. Vor einiger Zeit habe sie die Tante zu einem Geigenkonzert im Saal des neuen Sekundarschulhauses in Interlaken mitgenommen und nachher der Künstlerin vorgestellt. Man sei lange zusammen geblieben und erst nach Mitternacht ins Ried zurückgekommen; aber Fräulein Wach sei noch munter gewesen.

Wenn man auch einer unendlich bescheidenen Frau begegnete, gehörten doch starke Selbständigkeit und Eigenwille zu ihren Merkmalen. Sie hatte ihren Lebensstil bewahrt. Das Vorhandensein einer so ausgeprägten Persönlichkeit in unserer Gegenwart machte mir Eindruck.

Wenige Wochen nach unserem Besuch ist Marie Wach im Sommer 1964 gestorben. Ihr Grab liegt neben dem ihrer Eltern auf dem Gsteig-Friedhof in Wilderswil. Die Mendelssohn-Andenken haben die Schweiz verlassen. Die Stiftung Preußischer Kulturbesitz in Berlin hütet das Mendelssohn-Archiv. Das Album mit den Aquarellen werde ich kaum mehr sehen, aber nicht vergessen.

\section{Nachtrag}

Unerwartet steht im Jahrbuch vom Thuner- und Brienzersee 1982 (Spiez 1982), S.24-35, der Beitrag «Die Familie Mendelssohn und das Berner Oberland» von Redaktor Rudolf Wyß in Interlaken. Marie Wach wird gewürdigt und ihr Todestag angegeben (4.Juli 1964). - Ein Vergessener, der als schweizerischer Melodiespender Mendelssohn am nächsten trat, war der junge Herr Michel, der ihn auf der Fußreise 1831 von Unterssen an begleitet hat. Er wurde Wirt mit Familie in Meiringen. Nach frohem Wiedersehen über zehn Jahre später bat Mendelssohn brieflich Klingemann in London, Michels Aufnahme in John Murrays "Handbook for travellers in Switzerland" zu erwirken. 


\section{Summary}

This little sketeh is no contribution to the history of medicine. It only wants to give a portrait from memory. The object of the portrait is Marie Wach, a granddaughter of Felix Mendelssohn Bartholdy.

PI) Dr. phil. Heinz Balmer

Medizinhistorisches Institut

Rämistraße 71

CH-8006 Zürich 